\title{
Mycobacterium obuense Bacteremia in a Patient with Pneumonia
}

\section{Bruno Ali López Luis, Paulette Díaz-Lomelí, Livier Patricia Gómez-Albarrán, Areli Martínez-Gamboa, Alfredo Ponce-de-León}

Author affiliation: Instituto Nacional de Ciencias Médicas

y Nutrición Salvador Zubirán, Mexico City, Mexico

DOI: https://doi.org/10.3201/eid2505.180208

Mycobacterium obuense is a pigmented, rapidly growing mycobacterium. Because it has been considered nonpathogenic, $M$. obuense is being investigated in clinical trials of cancer immunotherapy and bioremediation. We report a case of bacteremia caused by $M$. obuense in a patient with pneumonia, showing its potential pathogenicity.

A pproximately 75 species of rapidly growing mycobacteria (RGM) have been isolated from soil, animals, and water (1). The RGM Mycobacterium obuense, an environmental pigmented mycobacterium, is mobile and easily adaptable to the environment and possesses oxygenases that enable it to degrade organic compounds and dechlorinate methoxychlor-based insecticides (2). Until recently, $M$. obuense has been considered nonpathogenic. We report a case of bacteremia caused by $M$. obuense.

A 29-year-old man from a rural community in Puebla, Mexico, arrived at an emergency department in Mexico City reporting a 2-day history of chest pain, dyspnea, and fever. On physical examination, his heart rate was $94 \mathrm{bpm}$, blood pressure 175/88 $\mathrm{mm} \mathrm{Hg}$, temperature $38.5^{\circ} \mathrm{C}$, and peripheral oxygen saturation $70 \%$. Chest auscultation revealed bibasilar fine crackles and signs of pleural effusion.

The patient was a farmer; had been in close contact with pigs, sheep, and cows; and reported consuming unpasteurized dairy products. He had a history of diabetes mellitus with chronic kidney disease categorized as stage G4 A3 (glomerular filtration rate $16.7 \mathrm{~mL} / \mathrm{min} / 1.73 \mathrm{~m}^{2}$; proteinuria $>2.8 \mathrm{~g} / \mathrm{d}$ ) of the KDIGO classification (Kidney Disease: Improving Global Outcomes, https://kdigo.org) without replacement therapy. He reported taking metformin, amlodipine, furosemide, and iron sulfate.

At admission, laboratory test results included leukocyte count, 11,900 cells $/ \mu \mathrm{L}$ with $88.2 \%$ neutrophils; Creactive protein, $250 \mathrm{mg} / \mathrm{L}$; procalcitonin, $17 \mathrm{ng} / \mathrm{mL}$; creatinine, $4.0 \mathrm{mg} / \mathrm{dL}$; and arterial blood gases, $\mathrm{pH} 7.24, \mathrm{pO}_{2}$ $40.8 \mathrm{~mm} \mathrm{Hg}, \mathrm{pCO}_{2} 34.8 \mathrm{~mm} \mathrm{Hg}$, lactate $2.9 \mathrm{mmol} / \mathrm{L}, \mathrm{HCO}_{3}$ $14.6 \mathrm{mmol} / \mathrm{L}$, and $\mathrm{sO}_{2} 71 \%$ on ambient air. Findings of a computed tomography scan of the chest suggested that the patient had a lung infection (Figure).

The patient began empirical treatment for communityacquired pneumonia with ceftriaxone and clarithromycin. No respiratory samples were obtained because the patient was unable to produce sputum. We performed blood cultures in Aerobic/F medium (Becton Dickinson, https:// www.bd.com). After a 7-day incubation period, we detected growth and observed gram-positive bacilli in the gram stain (Appendix Figure, panel A, http://wwwnc.cdc.gov/ EID/article/25/5/18-0208-App1.pdf). We made subcultures on sheep blood, chocolate, and Sabouraud agar and performed Kinyoun and Ziehl-Neelsen stains, in which we observed partially acidic alcohol-resistant coccobacilli (Appendix Figure, panels B,C). After 2 weeks of incubation at $35^{\circ}-37^{\circ} \mathrm{C}$, we observed rough, mucoid colonies (Appendix Figure, panels D,E).

We attempted to identify the bacilli by using GenoType Mycobacterium (Hain Lifescience, https://www. hain-lifescience.de) but were unsuccessful because no species-specific probe is available. We performed amplification and sequencing of the $16 \mathrm{~S}$ rRNA gene (498 bp) and hsp65 gene (400 bp). Pairwise sequence aligned 100\% with $16 \mathrm{~S}$ rRNA and $99.6 \%$ with $h s p 65$ to the sequences of M. obuense strain CIP 106803 (GenBank accession no. AF547954.1).

The patient was started on intravenous amikacin, clarithromycin, and moxifloxacin as soon as we notified clinicians of RGM isolated from his blood cultures. We conducted susceptibility testing by broth microdilution, which showed susceptibility to all antimicrobial drugs tested except tobramycin (intermediate susceptibility) (Appendix) (3).

The patient completed 2 weeks of intravenous moxifloxacin, amikacin, and clarithromycin and was discharged. He received 4 additional weeks of oral azithromycin and moxifloxacin and experienced complete resolution.

M. obuense, first described as a scotochromogenic RGM species isolated primarily from soil $>40$ years ago in Obu, Japan, is a catalase-positive, peroxidase-negative bacillus that can degrade salicylates, forming a black product $(4,5)$. In culture, $M$. obuense has 2 morphotypes, smooth and rough variants. In the smooth variants, its cell wall contains long-chain saturated fatty acids that enable it to colonize the environment and are responsible for the pleomorphism observed on the surface of solid agars ( 6 ). Although this species was later isolated from sputum samples from patients with apparent pulmonary disease, no additional clinical data were reported, and thus, no evidence of pathogenicity was established $(4,7)$.

Phylogenetic analysis of $M$. obuense shows close association with $M$. chubuense (81.3\% identity) and $M$. rufum (92.2\% identity) (7). Its genome consists of 5,576,960 bp 


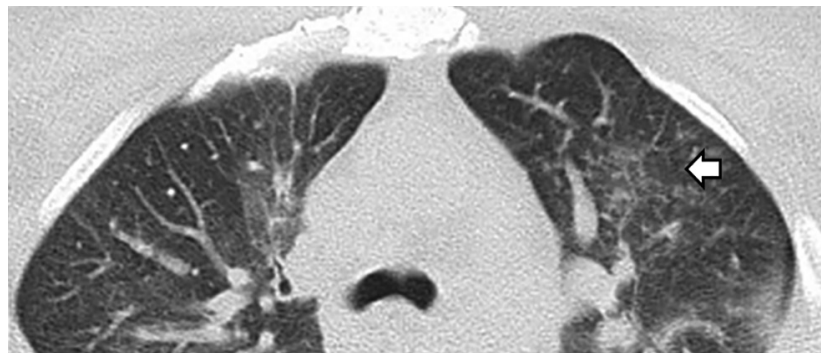

Figure. Computed tomography scan of the chest in a patient with Mycobacterium obuense pneumonia, Mexico, showing air space infiltration in the left parahilar and a tree bud pattern in the left upper lobe (arrow), as well as bilateral interstitial thickening and ground glass opacities.

(of which 133,713 bp are of plasmid origin with $68 \% \mathrm{GC}$ content) and 800 unique genes, more than related species, such as $M$. chubuense. Although these mycobacteria are considered nonpathogenic, they have homologous genes to mammalian cell entry that encode proteins involved in virulence and cell invasion. In addition, $M$. obuense contains genes involved in antimicrobial resistance, such as marA, aminoglycoside-resistance protein kinase, $\beta$-lactamases, and monooxygenases, which confer $M$. obuense with intrinsic rifampin resistance $(2,7)$.

Establishing etiology in this case was challenging because current nucleic acid probe assays cannot identify M. obuense correctly. Clinicians should avoid discarding RGM or misclassifying these isolates as colonizers until definitive species identification confirms etiology.

M. obuense has been evaluated as an adjuvant immunotherapy in phase I and II trials on patients with melanoma, pancreatic cancer, and colorectal cancer, with promising results. This treatment consists of an intradermal application of a suspension of heat-killed whole cell $M$. obuense (8-10).

The isolation of $M$. obuense from blood cultures of a patient with community-acquired pneumonia suggests its capacity for virulence and invasiveness in humans. Because $M$. obuense might become an adjuvant in cancer therapy, researchers should ensure implementation of proper, standardized inactivation protocols.

\section{About the Author}

Dr. Luis is a fellow in infectious diseases and Dr. Ponce-de-Leon is a senior researcher at the National Institute of Medical Science and Nutrition Salvador Zubiran, Mexico City. Their research fields are mycobacterial diseases and clinical microbiology.

\section{References}

1. Brown-Elliott BA, Philley JV. Rapidly growing mycobacteria. Microbiol Spectr. 2017;5. http://dx.doi.org/10.1128/ microbiolspec.TNMI7-0027-2016

2. Das S, Pettersson BM, Behra PR, Ramesh M, Dasgupta S, Bhattacharya A, et al. Characterization of three Mycobacterium spp. with potential use in bioremediation by genome sequencing and comparative genomics. Genome Biol Evol. 2015;7:1871-86. http://dx.doi.org/10.1093/gbe/evv111

3. Clinical and Laboratory Standards Institute. Susceptibility testing of Mycobacteria, Nocardiae, and other aerobic actinomycetes. Approved standard. 2nd edition. Document M24-A2. Wayne (PA): The Institute; 2011.

4. Tsukamura M, Mizuno S. Mycobacterium obuense, a rapidly growing scotochromogenic mycobacterium capable of forming a black product from $p$-aminosalicylate and salicylate. J Gen Microbiol. 1971;68:129-34. http://dx.doi.org/10.1099/ 00221287-68-2-129

5. Tsukamura M, Mizuno S, Tsukamura S. Numerical analysis of rapidly growing scotochromogenic mycobacteria, including Mycobacterium obuense sp. nov., nom. rev., Mycobacterium rhodesiae sp. nov., nom. rev., Mycobacterium aichiense sp. nov., nom. rev., Mycobacterium chubuense sp. nov., nom. rev., and Mycobacterium tokaiense sp. nov., nom. rev. Int J Syst Bacteriol. 1981;31:263-75. http://dx.doi.org/10.1099/ 00207713-31-3-263

6. Agustí G, Astola O, Rodríguez-Güell E, Julián E, Luquin M. Surface spreading motility shown by a group of phylogenetically related, rapidly growing pigmented mycobacteria suggests that motility is a common property of mycobacterial species but is restricted to smooth colonies. J Bacteriol. 2008;190:6894-902. http://dx.doi.org/10.1128/JB.00572-08

7. Greninger AL, Cunningham G, Hsu ED, Yu JM, Chiu CY, Miller S. Draft genome sequence of Mycobacterium obuense strain UC1, isolated from patient sputum. Genome Announc. 2015;3:e00612-15. http://dx.doi.org/10.1128/genomeA. 00612-15

8. Fowler D, Dalgleish A, Liu W. A heat-killed preparation of Mycobacterium obuense can reduce metastatic burden in vivo. J Immunother Cancer. 2014;2(Suppl 3):P54. http://dx.doi.org/ 10.1186/2051-1426-2-S3-P54

9. Stebbing J, Dalgleish A, Gifford-Moore A, Martin A, Gleeson C, Wilson $\mathrm{G}$, et al. An intra-patient placebo-controlled phase I trial to evaluate the safety and tolerability of intradermal IMM-101 in melanoma. Ann Oncol. 2012;23:1314-9. http://dx.doi.org/10.1093/ annonc/mdr363

10. Costa Neves M, Giakoustidis A, Stamp G, Gaya A, Mudan S. Extended survival after complete pathological response in metastatic pancreatic ductal adenocarcinoma following induction chemotherapy, chemoradiotherapy, and a novel immunotherapy agent, IMM-101. Cureus. 2015;7:e435.

Address for correspondence: Alfredo Ponce-de-León, Laboratory of Clinical Microbiology, Department of Infectious Diseases, Instituto Nacional de Ciencias Médicas y Nutrición Salvador Zubirán, Avenida Vasco de Quiroga No.15, Colonia Belisario Domínguez Sección XVI, Delegación Tlalpan, 14080, Mexico City, Mexico; email: alf.poncedeleon@gmail.com 